\section{BMJ Open}

Respiratory

Research

\title{
Regional variations in the prevalence and misdiagnosis of air flow obstruction in China: baseline results from a prospective cohort of the China Kadoorie Biobank (CKB)
}

Om P Kurmi, ${ }^{1}$ Liming Li, ${ }^{2,3}$ Margaret Smith, ${ }^{1}$ Mareli Augustyn, ${ }^{1}$ Junshi Chen, ${ }^{4}$ Rory Collins, ${ }^{1}$ Yu Guo, ${ }^{2}$ Yabin Han, ${ }^{5}$ Jingxin Qin, ${ }^{6}$ Guanqun Xu, ${ }^{7}$ Jian Wang, ${ }^{8}$ Zheng Bian, ${ }^{2}$ Gang Zhou, ${ }^{9}$ Kourtney Davis, ${ }^{10}$ Richard Peto, ${ }^{1}$ Zhenming Chen, ${ }^{1}$ on behalf of the China Kadoorie Biobank Collaborative Group

\section{ABSTRACT}

Smith M, et al. Regional variations in the prevalence and misdiagnosis of air flow obstruction in China: baseline results from a prospective cohort of the China Kadoorie Biobank (CKB). BMJ Open Resp Res 2014;1:e000025. doi:10.1136/bmjresp-2014000025

- Additional data are published online only. To view this file please visit the journal online (http://dx.doi. org/10.1136/bmjresp-2014000025)

Received 6 February 2014 Revised 14 April 2014 Accepted 16 April 2014

CrossMark

For numbered affiliations see end of article.

Correspondence to Dr Om P Kurmi; om.kurmi@ndph.ox.ac.uk
Background: Despite the great burden of chronic respiratory diseases in China, few large multicentre, spirometry-based studies have examined its prevalence, rate of underdiagnosis regionally or the relevance of socioeconomic and lifestyle factors.

Methods: We analysed data from 512891 adults in the China Kadoorie Biobank, recruited from 10 diverse regions of China during 2004-2008. Air flow obstruction (AFO) was defined by the lower limit of normal criteria based on spirometry-measured lung function. The prevalence of AFO was analysed by region, age, socioeconomic status, body mass index (BMI) and smoking history and compared with the prevalence of self-reported physician-diagnosed chronic bronchitis or emphysema (CB/E) and its symptoms.

Findings: The prevalence of AFO was $7.3 \%$ in men (range 2.5-18.2\%) and 6.4\% in women (1.5-18.5\%). Higher prevalence of AFO was associated with older age $(p<0.0001)$, lower income $(p<0.0001)$, poor education $(p<0.001)$, living in rural regions $(p<0.001)$, those who started smoking before the age of 20 years $(p<0.001)$ and low BMI $(p<0.001)$. Compared with self-reported diagnosis of $\mathrm{CB} / \mathrm{E}, 88.8 \%$ of $\mathrm{AFO}$ was underdiagnosed; underdiagnosis proportion was highest in 30-39-year olds $(96.7 \%)$ compared with the $70+$ age group $(81.1 \%)$, in women $(90.7 \%)$, in urban areas $(89.4 \%)$, in people earning $5 \mathrm{~K}-10 \mathrm{~K} ¥$ monthly $(90.3 \%)$ and in those with middle or high school education (92.6\%). Interpretation: In China, the burden of AFO based on spirometry was high and significantly greater than that estimated based on self-reported physician-diagnosed $\mathrm{CB} / \mathrm{E}$, especially in rural areas, reflecting major issues with diagnosis of AFO that will impact disease treatment and management.

\section{INTRODUCTION}

Globally, chronic obstructive pulmonary disease (COPD) is responsible for about

\section{KEY MESSAGES}

This is the largest population-based multi-centre study of prevalence and socioeconomic and lifestyles correlates of air flow obstruction (AFO) representative of adult Chinese population selected from ten diverse regions of China.

- The data suggests up to 10-fold difference in prevalence of AFO between different regions in China for both men and women.

- The result highlights that mis-diagnosis of AFO $(>80 \%)$ in Chinese population is a major issue requiring immediate attention to improve both appropriate management and prevention programs.

three million annual deaths, and for an even greater burden from disability, ${ }^{1}$ with particularly high-disease prevalence in low-income and middle-income countries such as China where smoking prevalence is very high among men. ${ }^{2}$ In China, over $90 \%$ of 1.4 million respiratory-related deaths ${ }^{3}$ and 10.4 million disability-adjusted life years ${ }^{4}$ are attributed to COPD in adults, with most of the COPD-related deaths occurring at the age of 60 years or older. ${ }^{4}$ Among published epidemiological studies conducted in China, there are large unexplained variations in the age-specific rates of COPD between men and women and between different regions, with reported prevalence ranging from 3\% to $12 \%$ in ages above 40 years. $^{5-7}$

Smoking is a major risk factor for COPD but few women in China smoke $(<5 \%)$, so this exposure cannot explain the relatively high prevalence of COPD seen in many parts of China. ${ }^{8}$ There is also evidence that 
exposure to environmental air pollutants particularly coal and wood smoke for cooking and heating, low socioeconomic status and lung infections such as tuberculosis earlier in life may contribute to increased risk of COPD, but the evidence is still extremely limited in China. ${ }^{5}$ As well as risk exposures, difference in survey methods and COPD diagnosis methods between different studies could also affect the burden of the disease estimated for different populations. There is good evidence that defining COPD based only on self-reported physician-diagnosis tends to significantly underestimate the true burden, particularly in resource-poor areas where access to healthcare is limited and also possibly due to lack of awareness of their condition. ${ }^{9}$ Despite this, most of the previous studies in China tended to use self-reported information rather than spirometry-defined COPD. Consequently, substantial uncertainty remains about the true burden of COPD in the population.

To help fill the gap in knowledge, we analysed the cross-sectional data of the China Kadoorie Biobank (CKB) of over 0.5 million adults from 10 diverse regions of China. ${ }^{10}$ The aims of the study were (1) to estimate the prevalence of air flow obstruction (AFO) based on the measured lung function and its variation with socioeconomic and lifestyle factors, (2) to examine the prevalence of self-reported physician-diagnosed chronic bronchitis/emphysema $(\mathrm{CB} / \mathrm{E})$, rates of treatment and (3) to assess the proportion of underdiagnosis by comparing the prevalence of AFO based on spirometry with self-reported physician-diagnosed $\mathrm{CB} / \mathrm{E}$ and any variation with socioeconomic and lifestyle factors.

\section{METHODS}

\section{Study design and participants}

A detailed description of the study design, survey methods and characteristics of participants for the CKB prospective study is published elsewhere. ${ }^{8}{ }^{10}$ In brief, the baseline survey took place between 2004 and 2008 involving five rural and five urban regions, chosen according to local disease patterns, exposure to certain risk factors, population stability, quality of death and diseases registries, local commitment and capacity. Overall, a total of 512891 adults (210 222 men and 302669 women) aged 30-79 were enrolled. All participants gave informed written consent.

\section{Data collection}

Laptop-based questionnaire was administered to each participant by trained health workers, who collected detailed information on demographic and socioeconomic status, dietary and other lifestyle factors (eg, smoking, alcohol use), exposure to passive smoking and household air pollution, respiratory symptoms (eg, chronic cough, production of chronic phlegm, breathlessness and severity of breathlessness), medical history of physician-diagnosed respiratory (chronic bronchitis, emphysema, asthma, tuberculosis) and other conditions (eg, stroke, ischaemic heart disease, cancer and diabetes), physical activity, sleeping and mental status and reproductive history (for women) at baseline. A range of physical measurements was taken, including standing and sitting height, weight, bioimpedance, exhaled carbon-monoxide and blood pressure.

\section{Spirometry and diagnostic criteria for AFO}

Spirometry was carried out by trained health technicians, using portable handheld 'Micro spirometer' (Micro Medical Limited, Rochester, Kent, England) in accordance with modified American Thoracic Society (ATS) ${ }^{11}$ procedures developed by our respiratory team. The spirometer we used during the baseline survey did not display flow volume loops, and hence the acceptability criterion of blows was modified. Participants made some practice blows, after which the results of two successful manoeuvres (as judged by the technician) were recorded for each participant. The larger of the two forced expiratory volume in $1 \mathrm{~s}\left(\mathrm{FEV}_{1}\right)$ and forced vital capacity (FVC) were used for calculating $\mathrm{FEV}_{1} / \mathrm{FVC}$ ratio and for further analysis. No bronchodilators were used at the baseline survey. Overall, 202 men and 194 women with an $\mathrm{FEV}_{1} /$ FVC $>1$ were excluded, leaving 210020 (99.9\%) men and 302475 (99.9\%) women for the present analysis.

For the present analysis, AFO is defined according to the lower limit of normal (LLN) definition as $\mathrm{FEV}_{1} / \mathrm{FVC}$ $<$ LLN. Values for the LLN were obtained from the Global Lung Initiative (GLI) reference equations for southeast Asian and northeast Asian population. ${ }^{12}$ The prevalence of modified restrictive abnormality, defined as an $\mathrm{FEV}_{1} / \mathrm{FVC} \geq \mathrm{LLN}$ and $\mathrm{FVC}<\mathrm{LLN}$, was also calculated, with LLN estimates also estimated by the GLI reference equations. ${ }^{12}$ For comparability with previous published studies, we also report AFO based on the Global Initiative for Obstructive Lung Disease (GOLD) criterion $\left(\mathrm{FEV}_{1} / \mathrm{FVC}<0.7\right)$, but without postbronchodilator lung function indices.

We defined chronic bronchitis as the presence of cough and phlegm for more than 3 months in the past 12 months. Underdiagnosis was defined as participants with AFO defined by spirometry but not physiciandiagnosed $\mathrm{CB} / \mathrm{E}$ and overdiagnosis was defined as those participants with physician-diagnosed $\mathrm{CB} / \mathrm{E}$ but not AFO defined by spirometry.

\section{Statistical methods}

All analyses were conducted separately for men and women. Baseline demographic characteristics were calculated by rural/urban area, and crude prevalence of AFO was calculated by region and urban/rural area. The prevalence of AFO (directly standardised to the study population male or female 5-year age group structure) was calculated for each region. Further, AFO prevalence for strata of various potential risk factors or correlates within urban and rural areas was calculated (directly standardised to the study population 10-year age group and region structure, as necessary). Similarly, 
we also calculated age and region-standardised prevalence of a number of chronic health conditions among those with AFO. Association between participants' characteristics and AFO diagnosis type was carried out using multivariate logistic regression. All statistical analyses were performed using SAS V.9.3.

\section{RESULTS}

At baseline, the overall mean age of participants was 52.0 \pm 10.7 years, $59 \%$ were women and $56 \%$ were from rural areas (table 1). The proportion of participants having at least 6 years of formal education was higher in urban compared with rural areas and higher in men than in women. The prevalence of ever regular smoking was significantly higher among men than women $(74.3 \%$ vs $3.2 \%)$ and somewhat higher in rural than in urban men $(77.7 \%$ vs $70.1 \%)$. The proportion reporting current use of clean fuel (ie, gas or electricity for cooking) was much higher in urban than in rural areas for men $(56.5 \%$ vs $7.1 \%)$ and women $(83.6 \%$ vs $12.4 \%)$. The mean body mass index (BMI) was lower in rural than in urban areas for men $\left(24.3 \mathrm{vs} 22.7 \mathrm{~kg} / \mathrm{m}^{2}\right)$ and women $\left(24.3 \mathrm{vs} 23.5 \mathrm{~kg} / \mathrm{m}^{2}\right)$, with approximately $5-6 \%$ of rural participants classified as underweight (BMI $<18.5 \mathrm{~kg} / \mathrm{m}^{2}$ ) compared with around $3 \%$ of urban participants (table 1 ).

The lung function indices $\left(\mathrm{FEV}_{1}, \mathrm{FVC}\right.$ and $\left.\mathrm{FEV}_{1} / \mathrm{FVC}\right)$ decreased steeply with increasing age (see online supplementary figures S1-S3) and were lower in rural than urban areas for men and women at all age groups (data not shown). Among women and men, ever smokers had higher $\mathrm{FEV}_{1}$ until the mid-40s; whereas FVC continued to be higher until the $50 \mathrm{~s}$, but then $\mathrm{FEV}_{1}$ and $\mathrm{FVC}$ decreased steeply with increasing age and were lower in ever smokers compared with never smokers.

Overall at baseline, $4.1 \%$ reported having chronic cough and phlegm, which was higher in rural than in urban areas for men $(7.3 \%$ vs $6.2 \%, \mathrm{p}<0.001)$ and women $(2.6 \%$ vs $1.8 \%, \mathrm{p}<0.001)$. Similar rural and urban differences were seen for breathlessness while walking on level ground for men $(5.8 \%$ vs $3.4 \%$, $\mathrm{p}<0.001)$ and women $(8.5 \%$ vs $4.7 \%, \mathrm{p}<0.001)$, but the reported prevalence was higher in women (table 2).

The prevalence of AFO (based on LLN) was higher in rural than urban areas for men $(9.2 \%$ vs $4.8 \%, \mathrm{p}<0.001)$ and women $(7.7 \%$ vs $4.8 \%, \mathrm{p}<0.001)$. Similar patterns were observed when AFO was based on fixed ratio criterion (GOLD grade 1+), and the overall prevalence was slightly lower compared with LLN $\left(\mathrm{FEV}_{1} / \mathrm{FVC}\right)$ except for those aged $>60$ years (figure 1 , table 3 and see online supplementary table S2). Regardless of the different definitions used, there was a nearly 10 -fold variation in the prevalence of AFO across the 10 study regions, with the highest prevalence observed in Sichuan (18.2\% vs $18.5 \%)$ and lowest in Harbin province (2.5\% vs $1.5 \%$ ) for men and women (figure 1, see online supplementary table S1). Age-adjusted prevalence based on GOLD grade 2+ was lower than LLN $\left(\mathrm{FEV}_{1} / \mathrm{FVC}\right)$ estimates in rural and urban men and women (figure 1, see online supplementary table S3). Similarly, the prevalence of AFO (adjusted for region) increased sharply among smokers particularly after the age of 50 (figure 2 and see online supplementary table $\mathrm{S} 4$ ). The prevalence of AFO among rural men and women increased significantly with age, exposure to wood or coal smoke while cooking, initiation of smoking at a younger age (under 20 years), ex-smokers who stopped smoking due to ill health and BMI $<18.5 \mathrm{~kg} / \mathrm{m}^{2}$, while AFO decreased with higher annual income and education (table 3, see online supplementary tables S2 and S3).

The prevalence of chronic bronchitis was somewhat greater in rural than in urban areas for men $(7.3 \%$ vs $6.2 \%)$ and women $(2.6 \%$ vs $1.8 \%)$. The prevalence of self-reported physician-diagnosed $\mathrm{CB} / \mathrm{E}$ was lower and approximately the same in the rural and urban areas for men $(3.2 \%$ vs $2.9 \%)$ and women $(2.1 \%$ vs $2.4 \%)$, among whom less than one-third reported currently taking medication for the condition. Around $80 \%$ of rural and $71 \%$ of urban men who reported a prior diagnosis of $\mathrm{CB} / \mathrm{E}$ were regular smokers and also reported chronic cough or breathlessness.

In most regions, the prevalence of $\mathrm{CB} / \mathrm{E}$ was lower than that of AFO diagnosed by spirometry. Of participants with prior physician diagnosis of $\mathrm{CB} / \mathrm{E}, 29.2 \%$ and $28.0 \%$ had AFO based on LLN and GOLD criteria, respectively (figure 3 and supplementary figure S4). The overall underdiagnosis proportion of AFO was $88.8 \%$, higher in urban than in rural areas $(89.4 \%$ vs $88.4 \%)$ and higher in women compared with men (90.7\% vs $86.2 \%)$. Similarly, the AFO overdiagnosis proportion was slightly higher in urban than in rural areas (81\% vs $62.2 \%)$ and higher in women compared with men (73.7\% vs $67.7 \%$ ) (table 4 ). Of those classified as AFO by spirometry, only $11.2 \%$ were correctly diagnosed previously by the physician. The underdiagnosis proportion of AFO was higher in those with lower household income, younger age, having less chronic respiratory symptoms, women, in current regular smokers, but lower in ex-smokers (see online supplementary table S4). There was wide regional variation in the underdiagnosis and overdiagnosis proportion, as well as variation in the treatment for physician-diagnosed $\mathrm{CB} / \mathrm{E}$ cases (see online supplementary table S1 and figure S5). Sichuan, with the highest prevalence of AFO, had the lowest percentages of overdiagnosed $(<40 \%)$ AFO. Patterns similar to underdiagnosis were observed for overdiagnosis as well, except it was lower in women. There was also a lack of concordance between self-reported symptom-based chronic bronchitis and spirometry-based AFO (table 3, see online supplementary table S1 and figure 4 , see online supplementary figure $\mathbf{S 6}$ ).

The prevalence of self-reported doctor-diagnosed asthma was $<1 \%$ among men and women, whereas tuberculosis was marginally greater in urban than in rural areas for men $(2.6 \%$ vs $1.6 \%)$ and women $(1.5 \%$ vs $0.8 \%)$. The prevalence of restrictive abnormality was highest among the ex-regular smokers (men vs women: 
Table 1 Baseline characteristics of participants by sex and region types (figures in the column are $\%$ of total)

\begin{tabular}{|c|c|c|c|c|}
\hline \multirow[b]{2}{*}{ Characteristics } & \multicolumn{2}{|l|}{ Men } & \multicolumn{2}{|l|}{ Women } \\
\hline & $\begin{array}{l}\text { Rural (\%) } \\
\mathrm{N}=118837\end{array}$ & $\begin{array}{l}\text { Urban (\%) } \\
\mathrm{N}=91220\end{array}$ & $\begin{array}{l}\text { Rural (\%) } \\
\mathrm{N}=167727\end{array}$ & $\begin{array}{l}\text { Urban (\%) } \\
\mathrm{N}=134711\end{array}$ \\
\hline Height (mean \pm SD in $\mathrm{cm})$ & $164.1 \pm 6.3$ & $166.8 \pm 6.5$ & $153.2 \pm 5.9$ & $155.3 \pm 5.9$ \\
\hline \multicolumn{5}{|l|}{ Age (years) } \\
\hline $30-39$ & 14.5 & 13.5 & 18.2 & 13.1 \\
\hline $40-49$ & 27.7 & 28.8 & 31.4 & 30.2 \\
\hline $50-59$ & 31.2 & 29.1 & 31.2 & 30.7 \\
\hline $60-69$ & 19.8 & 19.5 & 15.0 & 18.8 \\
\hline $70-79$ & 6.8 & 9.1 & 4.2 & 7.2 \\
\hline Mean (SE) & $52.6(0.03)$ & $53.1(0.04)$ & $50.5(0.02)$ & $52.6(0.03)$ \\
\hline \multicolumn{5}{|l|}{ BMI $\left(\mathrm{kg} / \mathrm{m}^{2}\right)$} \\
\hline$<18.5$ & 5.7 & 2.8 & 5.2 & 3.2 \\
\hline 18.5 to $<25$ & 71.5 & 55.3 & 64.3 & 57.9 \\
\hline$\geq 25$ & 22.7 & 41.8 & 30.5 & 38.9 \\
\hline Mean (SE) & $22.7(0.01)$ & $24.3(0.01)$ & $23.5(0.01)$ & $24.3(0.01)$ \\
\hline \multicolumn{5}{|l|}{ Smoking status } \\
\hline Never smoker & 11.3 & 18.4 & 94.4 & 95.5 \\
\hline Occasional smoker & 11.0 & 11.5 & 2.0 & 1.6 \\
\hline Ex-regular smoker & 11.8 & 15.3 & 0.9 & 0.8 \\
\hline Current regular smoker & 65.9 & 54.8 & 2.6 & 2.1 \\
\hline \multicolumn{5}{|l|}{ Pack years $^{*}$} \\
\hline$<10$ & 19.7 & 19.3 & 47.7 & 51.7 \\
\hline $10-20$ & 23.1 & 26.3 & 26.5 & 25.1 \\
\hline$>20$ & 57.1 & 54.5 & 25.8 & 23.2 \\
\hline Mean (SE) & $26.7(0.07)$ & $24.8(0.07)$ & $15.2(0.2)$ & $13.7(0.22)$ \\
\hline \multicolumn{5}{|l|}{ Age started smoking (years) } \\
\hline$<20$ & 34.7 & 33.1 & 35.1 & 21.9 \\
\hline $20-24$ & 38.0 & 36.6 & 21.4 & 16.6 \\
\hline $25-29$ & 12.5 & 15.8 & 12.2 & 12.4 \\
\hline$\geq 30$ & 14.8 & 14.6 & 31.4 & 49.1 \\
\hline \multicolumn{5}{|c|}{ Number of cigarettes smoked daily (or equivalent) } \\
\hline $1-4$ & 7.2 & 4.7 & 29.1 & 24.9 \\
\hline $5-14$ & 27.5 & 30.8 & 47.2 & 51.3 \\
\hline $15-24$ & 45.1 & 47.8 & 20.4 & 20.7 \\
\hline$\geq 25$ & 20.2 & 16.7 & 3.3 & 3.1 \\
\hline \multicolumn{5}{|l|}{ Reason for stopping among ex-smokers } \\
\hline Physical illness & 53.2 & 45.9 & 64.1 & 40.8 \\
\hline Other reason & 46.8 & 54.1 & 35.9 & 59.2 \\
\hline Exposure to passive smoking $\dagger$ & 73.7 & 63.0 & 87.5 & 83.2 \\
\hline \multicolumn{5}{|l|}{ Highest education completed } \\
\hline No formal education & 12.7 & 4.0 & 31.7 & 17.3 \\
\hline Primary school & 43.8 & 19.7 & 40.4 & 20.3 \\
\hline Middle or high school & 41.8 & 60.5 & 27.2 & 53.3 \\
\hline College or university & 1.7 & 15.9 & 0.6 & 9.2 \\
\hline \multicolumn{5}{|l|}{ Household income (yuan/year) } \\
\hline 2500-4999 & 14.3 & 2.6 & 15.1 & 4.1 \\
\hline 5000-9999 & 23.5 & 8.0 & 26.1 & 11.5 \\
\hline 10 000-19999 & 28.9 & 27.6 & 28.9 & 30.4 \\
\hline$\geq 20000$ & 33.3 & 61.7 & 29.9 & 54.1 \\
\hline \multicolumn{5}{|l|}{ Exposure to cooking fuelsł } \\
\hline Currently cooks with coal/wood & 27.7 & 5.3 & 81.0 & 8.7 \\
\hline Ever cooked with coal/wood & 32.7 & 33.6 & 92.6 & 61.0 \\
\hline Currently cooks with gas/electricity & 7.1 & 56.5 & 12.4 & 83.6 \\
\hline \multicolumn{5}{|l|}{ Respiratory symptoms } \\
\hline Chronic cough and phlegm & 7.3 & 6.2 & 2.6 & 1.8 \\
\hline Breathlessness & 5.8 & 3.4 & 8.5 & 4.7 \\
\hline
\end{tabular}

${ }^{*}$ Restricted to ever regular smokers.

†Defined as never smokers who lived with a smoker or were exposed at work for 1-5 days/week or daily. †Restricted to participants who reported cooking daily or weekly.

$\mathrm{BMI}$, body mass index. 
Table 2 Participant characteristics relating to AFO, by region and sex (figures are in percentage of total unless stated)

\begin{tabular}{|c|c|c|c|c|c|c|}
\hline & \multicolumn{3}{|l|}{ Men } & \multicolumn{3}{|l|}{ Women } \\
\hline & Rural & Urban & Overall & Rural & Urban & Overall \\
\hline Total & 118837 & 91220 & 210057 & 167727 & 134711 & 302438 \\
\hline Mean age (years) & 52.6 & 53.1 & 52.9 & 50.5 & 52.6 & 51.5 \\
\hline AFO GOLD stage I-IV & 8.4 & 4.5 & 6.7 & 5.5 & 3.3 & 4.4 \\
\hline AFO GOLD stage II-IV & 7.0 & 3.6 & 5.5 & 4.6 & 2.4 & 3.5 \\
\hline AFO LLN & 9.2 & 4.8 & 7.3 & 7.7 & 4.8 & 6.3 \\
\hline \multicolumn{7}{|c|}{ Classification of severity of AFO (values are \% predicted)* } \\
\hline Mild $\left(\mathrm{FEV}_{1} \geq 80 \%\right)$ & 1.4 & 0.9 & 1.2 & 0.9 & 0.9 & 1.4 \\
\hline Moderate $\left(50 \% \leq \mathrm{FEV}_{1} \geq 80 \%\right)$ & 4.2 & 2.4 & 3.4 & 2.9 & 1.7 & 4.2 \\
\hline Severe $\left(50 \% \leq \mathrm{FEV}_{1} \geq 80 \%\right)$ & 2.2 & 1.0 & 1.6 & 1.4 & 0.6 & 2.2 \\
\hline Very severe $\left(\mathrm{FEV}_{1}<80 \%\right)$ & 0.7 & 0.3 & 0.5 & 0.3 & 0.1 & 0.7 \\
\hline Doctor diagnosed CB/E & 3.2 & 2.9 & 3.1 & 2.1 & 2.4 & 2.2 \\
\hline Doctor diagnosed CB/E and still on treatment & 34.8 & 28.4 & 32.1 & 37.8 & 27.4 & 32.7 \\
\hline \multicolumn{7}{|l|}{ Doctor diagnosed CB/E and } \\
\hline AFO GOLD stage I-IV† & 40.5 & 22.8 & 33.1 & 31.5 & 14.3 & 23.0 \\
\hline AFO GOLD stage II-IV† & 39.6 & 21.7 & 32.2 & 30.4 & 12.9 & 21.7 \\
\hline AFO LLN† & 39.5 & 22.0 & 32.3 & 35.6 & 16.7 & 26.3 \\
\hline Under diagnosis & 86.1 & 86.6 & 86.2 & 90.3 & 91.5 & 90.7 \\
\hline Over diagnosis & 60.5 & 78.0 & 67.7 & 64.4 & 83.3 & 73.7 \\
\hline Doctor diagnosed asthma & 0.4 & 0.8 & 0.6 & 0.3 & 0.8 & 0.5 \\
\hline Breathlessness $\ddagger$ & 5.8 & 3.4 & 4.7 & 8.5 & 4.7 & 6.8 \\
\hline Chronic cough§ & 6.8 & 4.5 & 5.8 & 3.0 & 1.8 & 2.4 \\
\hline Chronic cough and sputum & 7.3 & 6.2 & 6.8 & 2.6 & 1.8 & 2.2 \\
\hline \multicolumn{7}{|l|}{ Chronic cough with sputum and } \\
\hline AFO GOLD stage I-IV** & 17.3 & 9.8 & 14.4 & 17.5 & 8.4 & 14.1 \\
\hline AFO GOLD stage $\|-I V^{* *}$ & 15.8 & 8.6 & 13.1 & 15.8 & 7.2 & 12.7 \\
\hline AFO LLN** & 18.9 & 10.1 & 15.5 & 20.8 & 10.0 & 16.9 \\
\hline
\end{tabular}

$p$ Value for difference between urban and rural $<0.0001$ except men: doctor-diagnosed $C B / E(p=0.0002)$; underdiagnosis $(p=0.3775)$ women: underdiagnosis $(\mathrm{p}=0.0032)$.

${ }^{*}$ Based on prebronchodilator $\mathrm{FEV}_{1}$ in participants with $\mathrm{FEV}_{1} / \mathrm{FVC}<0.70$ according to modified GOLD definition.

†Figures are percentage of different GOLD stages of AFO or LLN based AFO in those with doctor diagnosed CB/E.

‡Becomes short of breath while walking on level ground with healthy people of same age.

$\S$ Had cough for at least 3 months in the past 12 months.

ๆCough up sputum in the morning for at least 3 months in the past 12 months.

${ }^{* \star}$ Figures are percentage of different GOLD stages of AFO or LLN based AFO in those with chronic cough and sputum.

AFO, air flow obstruction; CB/E, chronic bronchitis/emphysema; FEV ${ }_{1}$, forced expiratory volume in $1 \mathrm{~s}$; GOLD, Global Initiative for Obstructive Lung Disease; LLN, lower limit of normal.

Figure 1 Prevalence of air flow obstruction (age-adjusted) by sex and region.

\section{Age-adjusted prevalence of AFO by sex and region \\ Males \\ Females}
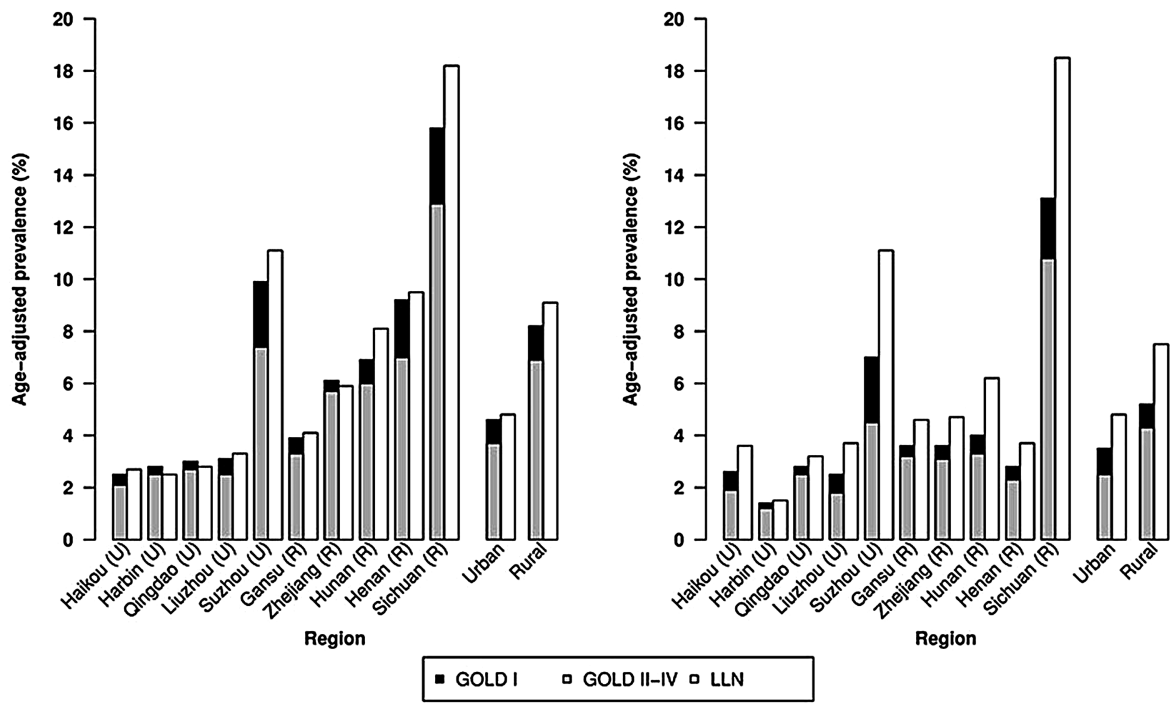
Table 3 Age and region standardised prevalence of AFO based on LLN definition, by patient characteristics

\begin{tabular}{|c|c|c|c|c|c|c|c|c|}
\hline \multirow[b]{3}{*}{ Characteristics } & \multicolumn{4}{|l|}{ Men } & \multicolumn{4}{|l|}{ Women } \\
\hline & \multicolumn{2}{|l|}{ Rural } & \multicolumn{2}{|l|}{ Urban } & \multicolumn{2}{|l|}{ Rural } & \multicolumn{2}{|l|}{ Urban } \\
\hline & $\overline{\mathbf{N}}$ & Per cent & $\overline{\mathbf{N}}$ & Per cent & $\overline{\mathbf{N}}$ & Per cent & $\overline{\mathbf{N}}$ & Per cent \\
\hline Overall & 118837 & 9.2 & 91220 & 4.8 & 167727 & 7.7 & 134711 & 4.8 \\
\hline \multicolumn{9}{|l|}{ Age group (\%) } \\
\hline $30-39$ & 17204 & 6.2 & 12361 & 2.8 & 30478 & 6.9 & 17695 & 4.7 \\
\hline $40-49$ & 32932 & 6.3 & 26230 & 3.3 & 52743 & 5.6 & 40707 & 4.1 \\
\hline $50-59$ & 37100 & 8.7 & 26571 & 4.6 & 52371 & 7.1 & 41400 & 4.3 \\
\hline $60-69$ & 23544 & 12.9 & 17766 & 6.9 & 25137 & 10.4 & 25269 & 5.9 \\
\hline $70-79$ & 8057 & 16.8 & 8292 & 9.6 & 6998 & 15.3 & 9640 & 7.8 \\
\hline$p$ for trend & $<0.0001$ & & $<0.0001$ & & $<0.0001$ & & $<0.0001$ & \\
\hline \multicolumn{9}{|l|}{ BMl group (\%) } \\
\hline$<18.5$ & 6821 & 18.0 & 2599 & 10.8 & 8680 & 12.7 & 4259 & 7.7 \\
\hline $18.5-24$ & 85013 & 9.5 & 50482 & 5.5 & 107832 & 7.8 & 78023 & 5.3 \\
\hline$\geq 25$ & 27002 & 5.9 & 38139 & 3.4 & 51214 & 6.0 & 52429 & 3.9 \\
\hline$p$ for trend & $<0.0001$ & & $<0.0001$ & & $<0.0001$ & & $<0.0001$ & \\
\hline \multicolumn{9}{|l|}{ Smoking category (\%) } \\
\hline Never & 13486 & 7.8 & 16796 & 3.5 & 158401 & 7.3 & 128716 & 4.8 \\
\hline Occasional & 13123 & 7.6 & 10487 & 3.3 & 3389 & 7.8 & 2133 & 4.2 \\
\hline Ex-regular & 13980 & 9.7 & 13913 & 5.4 & 1561 & 7.8 & 1083 & 6.2 \\
\hline Current regular & 78248 & 9.5 & 50024 & 5.3 & 4376 & 9.8 & 2779 & 5.8 \\
\hline Ever regular & 92228 & 9.6 & 63937 & 5.4 & 5937 & 9.7 & 3862 & 5.7 \\
\hline$p$ for heterogeneity & $<0.0001$ & & $<0.0001$ & & $<0.0001$ & & $<0.0001$ & \\
\hline \multicolumn{9}{|l|}{ Pack years* ${ }^{*}$} \\
\hline$<10$ & 18211 & 8.4 & 12322 & 4.2 & 2831 & 10.0 & 1998 & 5.7 \\
\hline $10-19$ & 21324 & 8.8 & 16786 & 4.9 & 1574 & 11.0 & 968 & 5.1 \\
\hline$>20$ & 52693 & 10.4 & 34829 & 6.0 & 1532 & 13.8 & 896 & 9.0 \\
\hline$p$ for trend & $<0.0001$ & & $<0.0001$ & & 0.2402 & & 0.1224 & \\
\hline \multicolumn{9}{|c|}{ Age started smoking (years) } \\
\hline$<20$ & 32031 & 10.5 & 21159 & 6.6 & 2083 & 13.6 & 846 & 10.1 \\
\hline $20-24$ & 35021 & 9.7 & 23375 & 5.3 & 1268 & 14.0 & 643 & 5.4 \\
\hline $25-29$ & 11572 & 9.1 & 10097 & 5.0 & 724 & 8.9 & 477 & 5.2 \\
\hline$\geq 30$ & 13604 & 8.3 & 9306 & 4.5 & 1862 & 9.9 & 1896 & 5.2 \\
\hline$p$ for trend & $<0.0001$ & & $<0.0001$ & & $<0.0001$ & & $<0.0001$ & \\
\hline \multicolumn{9}{|c|}{ Number of cigarettes smoked daily (or equivalent) } \\
\hline $1-4$ & 6605 & 9.3 & 2992 & 4.6 & 1726 & 9.6 & 961 & 5.5 \\
\hline $5-14$ & 25376 & 9.2 & 19681 & 5.2 & 2805 & 11.3 & 1982 & 5.2 \\
\hline $15-24$ & 41631 & 9.8 & 30558 & 5.5 & 1212 & 12.1 & 801 & 6.8 \\
\hline$\geq 25$ & 18616 & 10.1 & 10706 & 5.8 & 194 & 8.9 & 118 & 5.5 \\
\hline$p$ for trend & 0.0019 & & 0.0227 & & 0.3553 & & 0.1128 & \\
\hline \multicolumn{9}{|c|}{ Reason for stopping among ex-smokers } \\
\hline Physical illness & 7442 & 13.2 & 6386 & 8.7 & 1001 & 31.8 & 442 & 6.7 \\
\hline Other reason & 6538 & 8.8 & 7527 & 4.4 & 560 & 21.9 & 641 & 4.5 \\
\hline$p$ for heterogeneity & $<0.0001$ & & $<0.0001$ & & $<0.0001$ & & 0.0918 & \\
\hline \multicolumn{9}{|l|}{ Passive smokingt } \\
\hline No & 3546 & 9.4 & 6211 & 4.1 & 19783 & 9.0 & 21643 & 4.8 \\
\hline Yes & 9940 & 7.4 & 10585 & 3.3 & 138618 & 7.1 & 107073 & 4.7 \\
\hline$p$ for heterogeneity & 0.0001 & & 0.1538 & & $<0.0001$ & & 0.3864 & \\
\hline Highest education com & ted & & & & & & & \\
\hline No formal education & 15043 & 12.0 & 3606 & 8.3 & 53238 & 9.9 & 23254 & 5.5 \\
\hline Primary school & 52103 & 10.0 & 17983 & 5.8 & 67726 & 7.4 & 27309 & 4.8 \\
\hline Middle/high school & 49621 & 7.6 & 55167 & 4.2 & 45690 & 6.3 & 71772 & 4.4 \\
\hline College/university & 2070 & 7.1 & 14464 & 4.3 & 1073 & 5.7 & 12376 & 3.9 \\
\hline $\mathrm{p}$ for trend & $<0.0001$ & & $<0.0001$ & & $<0.0001$ & & $<0.0001$ & \\
\hline Household income ( $¥)$ & & & & & & & & \\
\hline $2500-4999$ & 17022 & 12.9 & 2413 & 8.4 & 25256 & 10.5 & 5461 & 5.7 \\
\hline 5000-9999 & 27939 & 10.9 & 7319 & 6.2 & 43775 & 8.5 & 15521 & 5.4 \\
\hline $10000-19999$ & 34326 & 9.2 & 25184 & 5.4 & 48506 & 7.2 & 40887 & 5.2 \\
\hline$\geq 20000$ & 39550 & 6.7 & 56304 & 4.3 & 50190 & 6.1 & 72842 & 4.4 \\
\hline$p$ for trend & $<0.0001$ & & $<0.0001$ & & $<0.0001$ & & $<0.0001$ & \\
\hline
\end{tabular}


Table 3 Continued

\begin{tabular}{|c|c|c|c|c|c|c|c|c|}
\hline \multirow[b]{3}{*}{ Characteristics } & \multicolumn{4}{|l|}{ Men } & \multicolumn{4}{|l|}{ Women } \\
\hline & \multicolumn{2}{|l|}{ Rural } & \multicolumn{2}{|l|}{ Urban } & \multicolumn{2}{|l|}{ Rural } & \multicolumn{2}{|l|}{ Urban } \\
\hline & $\mathbf{N}$ & Per cent & $\mathbf{N}$ & Per cent & $\mathbf{N}$ & Per cent & $\mathbf{N}$ & Per cent \\
\hline \multicolumn{9}{|c|}{ Currently cooks with coal/wood } \\
\hline No & 85965 & 8.7 & 86428 & 4.7 & 31872 & 7.5 & 122950 & 4.8 \\
\hline Yes & 32872 & 10.1 & 4792 & 6.5 & 135855 & 7.6 & 11761 & 5.5 \\
\hline$p$ for heterogeneity & $<0.0001$ & & $<0.0001$ & & $<0.0001$ & & 0.7517 & \\
\hline \multicolumn{9}{|c|}{ Ever cooked with coal/wood } \\
\hline No & 80005 & 8.6 & 60591 & 4.6 & 12381 & 7.8 & 52523 & 4.6 \\
\hline Yes & 38832 & 10.0 & 30629 & 5.2 & 155346 & 7.6 & 82188 & 5.0 \\
\hline$p$ for heterogeneity & $<0.0001$ & & 0.0014 & & $<0.0001$ & & 0.0160 & \\
\hline
\end{tabular}

${ }^{*}$ Restricted to ever regular smokers.

†Among never smokers, exposed to others' tobacco smoke regularly at home or work.

$\mathrm{BMI}$, body mass index.

$29.5 \%$ vs $25.1 \%$ ), men aged $70-79$ years $(37.5 \%)$ and women aged $30-39$ years $(28.7 \%$; see online supplementary table S5).

\section{DISCUSSION}

This is by far the largest population-based study in China of the prevalence and socioeconomic and lifestyle correlates of AFO in adult Chinese men and women. It involved 10 geographically and socioeconomically diverse regions and showed that there is a wide heterogeneity in the prevalence of AFO by region, age, socioeconomic and lifestyle factors such as smoking and BMI. Overall, self-reported prior diagnosis of $\mathrm{CB} / \mathrm{E}$ was found to be poorly correlated with AFO based on the measured lung function and less than one-third of those

Figure 2 Prevalence of air flow Region-adjusted prevalence of AFO (LLN) obstruction (region-adjusted) by age group.
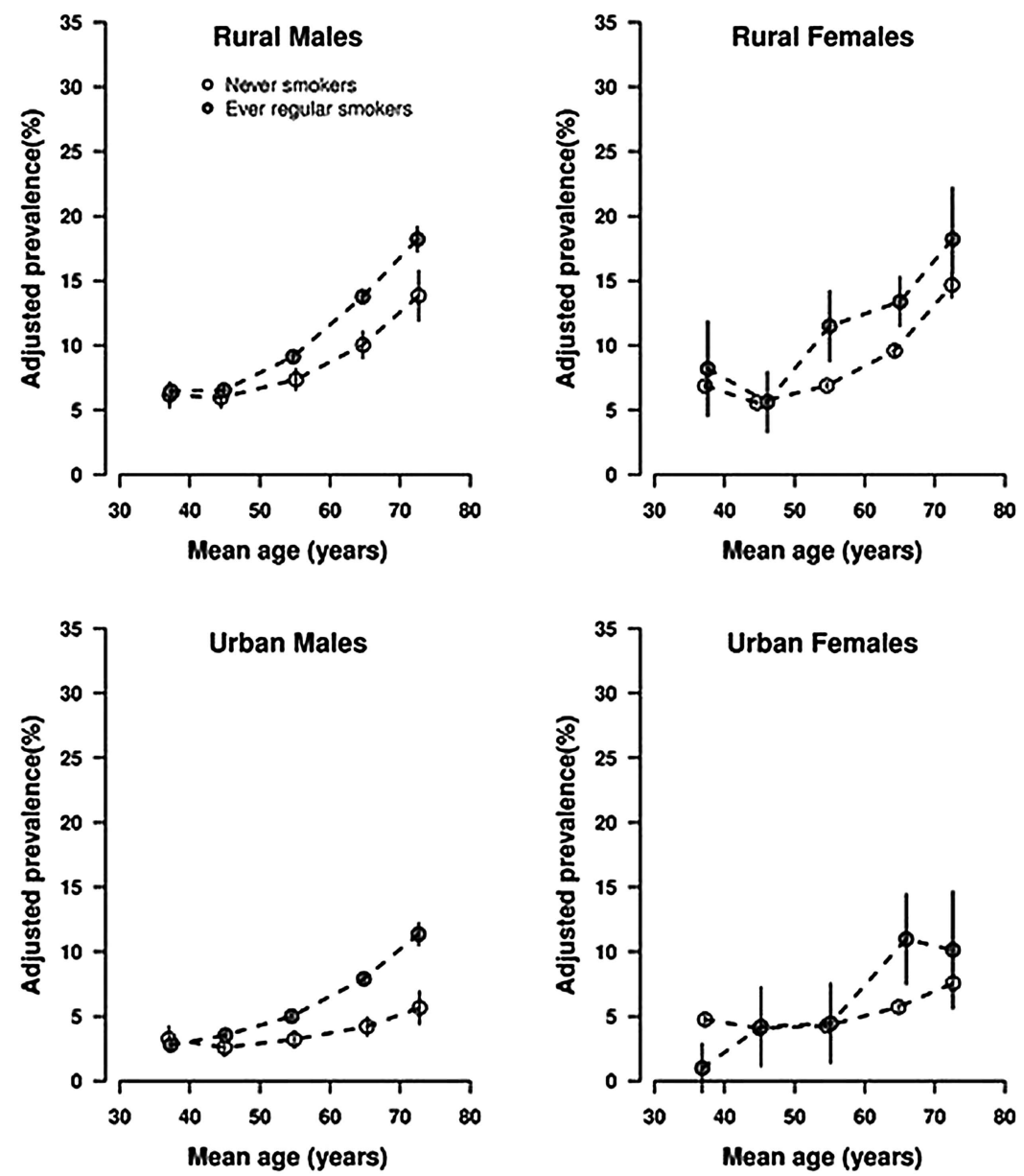
Table 4 Age and region-adjusted prevalence, stratified by various baseline variables

\begin{tabular}{|c|c|c|c|c|c|c|c|c|c|c|c|c|}
\hline \multirow[b]{2}{*}{ Characteristics } & \multicolumn{6}{|c|}{$\begin{array}{l}\text { Men (prevalence (\%), unless otherwise } \\
\text { stated) }\end{array}$} & \multicolumn{6}{|c|}{$\begin{array}{l}\text { Women (prevalence (\%) unless otherwise } \\
\text { stated) }\end{array}$} \\
\hline & $\mathbf{N}$ & CB/E & AFO & UD & OD & $\mathrm{CB} / \mathrm{E}_{\mathrm{T}}$ & $\mathbf{N}$ & CB/E & AFO & UD & OD & $\mathrm{CB} / \mathrm{E}_{\mathrm{T}}$ \\
\hline \multicolumn{13}{|l|}{ Sampling regions } \\
\hline Rural & 118837 & 3.2 & 9.2 & 86.1 & 60.5 & 34.8 & 167727 & 2.1 & 7.7 & 90.3 & 64.4 & 37.8 \\
\hline Urban & 91220 & 2.9 & 4.8 & 86.6 & 78.0 & 28.4 & 134711 & 2.4 & 4.8 & 91.5 & 83.3 & 27.4 \\
\hline 5000-9999 & 35258 & 3.5 & 8.9 & 85.9 & 61.8 & 33.3 & 59296 & 2.3 & 7.1 & 90.7 & 73.5 & 36.1 \\
\hline $10000-19999$ & 59510 & 3.0 & 7.5 & 86.7 & 67.4 & 31.9 & 89393 & 2.2 & 6.3 & 90.9 & 73.9 & 33.2 \\
\hline$\geq 20000$ & 95854 & 3.0 & 5.6 & 84.5 & 71.3 & 29.4 & 123032 & 2.3 & 5.3 & 89.3 & 78.1 & 29.0 \\
\hline \multicolumn{13}{|c|}{ Highest education completed } \\
\hline No formal education & 18649 & 3.1 & 10.4 & 85.5 & 54.4 & 32.1 & 76492 & 2.4 & 7.9 & 87.5 & 62.9 & 35.1 \\
\hline Never & 30282 & 3.3 & 5.9 & 83.8 & 70.3 & 32.2 & 287117 & 2.1 & 5.9 & 91.2 & 76.2 & 31.6 \\
\hline Occasional & 23610 & 3.1 & 5.7 & 88.1 & 75.4 & 27.6 & 5522 & 2.7 & 10.2 & 90.1 & 59.6 & 39.5 \\
\hline Ex-regular & 27893 & 5.6 & 7.8 & 73.9 & 61.7 & 38.6 & 2644 & 6.1 & 14.7 & 77.7 & 40.1 & 43.4 \\
\hline \multirow{2}{*}{\multicolumn{13}{|c|}{ Body mass index $\left(\mathrm{kg} / \mathrm{m}^{2}\right)$}} \\
\hline & & & & & & & & & & & & \\
\hline$<18.5$ & 9420 & 6.0 & 14.9 & 77.5 & 45.8 & 39.2 & 12939 & 4.4 & 10.5 & 83.4 & 59.9 & 36.4 \\
\hline 18.5 to $<25$ & 135495 & 2.9 & 7.8 & 87.1 & 66.4 & 31.7 & 185855 & 2.1 & 6.7 & 91.2 & 72.1 & 32.7 \\
\hline$\geq 25$ & 65141 & 3.0 & 4.8 & 86.4 & 78.3 & 28.6 & 103643 & 2.2 & 5.1 & 92.0 & 80.8 & 31.7 \\
\hline \multicolumn{13}{|l|}{ Age group (years) } \\
\hline $30-39$ & 29565 & 1.1 & 4.7 & 96.0 & 81.8 & 19.7 & 48173 & 1.0 & 5.9 & 96.8 & 80.5 & 22.7 \\
\hline $40-49$ & 59162 & 1.4 & 5.0 & 93.9 & 78.9 & 21.0 & 93450 & 1.4 & 4.9 & 94.8 & 81.0 & 25.0 \\
\hline
\end{tabular}

with physician diagnosis were receiving treatment at the time of the survey. The estimated underdiagnosis and overdiagnosis proportions were high in rural and urban areas.

Several studies from China have estimated COPD prevalence in adult populations, but the results have not been consistent, with the reported prevalence between $3 \%$ and $12 \% .^{5-7}$ In our study, the overall weighted prevalence of AFO based on GOLD 1+ definition for ages 4079 was $6 \%$ (ranging from $2 \%$ in Harbin to $14 \%$ in Sichuan), much lower than that reported by Buist et al for China $(11.4 \%)$ and many other countries such as the USA (19.6\%), Australia (19.2\%), Turkey (10.1\%), Austria $(26.1 \%)$, Iceland $(17.8 \%)$ and Poland $(22.1 \%)$. Although the study by Buist et al measured postbronchodilator lung function, the sample sizes were much smaller (473-893 in each country) than ours $(>0.5$ million) and the majority of them were from one region or city in each of the countries and thus, could not be nationally representative, particularly in a country with large heterogeneity such as China. Despite this, the large difference in the prevalence of AFO between CKB population and previous studies of Western and Chinese populations is likely to be largely real, for the CKB participants were much younger, with only $6.4 \%$ aged $\geq 70$ years compared with $10-25 \%$ participants in other studies. ${ }^{5}$ The huge variation in the reported prevalence from different countries and even in different or same regions of a country could be accounted for by several factors, including data acquisition methods, quality control of spirometry measurements, types of sampling population (such as exposure to environmental pollutants, age, previous history of diseases, smoking history and family history, dietary patterns and physical activity) as well as the diagnostic criteria used (eg, GOLD, ERS/ ATS criteria used to define COPD). ${ }^{13} 14$

Our study confirms the previous observations ${ }^{5}$ of large heterogeneity in the prevalence of AFO across different 

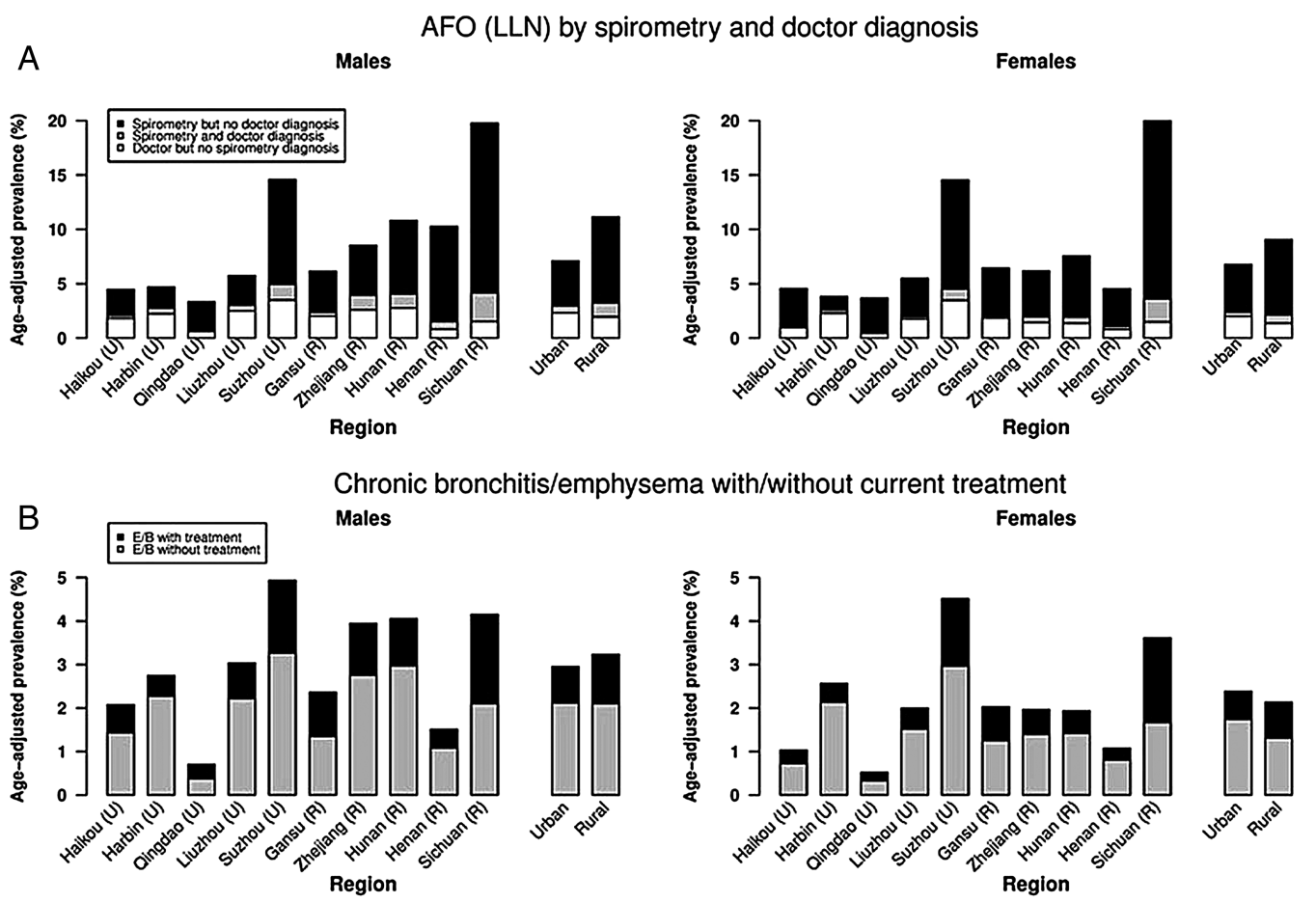

Figure 3 Prevalence of air flow obstruction (age-adjusted) by (A) lower limit of normal of forced expiratory ratio versus self-reported doctor diagnosis and (B) self-reported doctor diagnosis with/without current treatment.

regions of China, with very high prevalence in the Sichuan region for men and women. We did not compare the nutrient intake and physical activities across different regions in this paper, but hypothesise that it is highly unlikely that lifestyle factors could completely explain the substantial heterogeneity observed across different regions. Most of the rural participants were farmers and there were no major differences in the dietary patterns or smoking habit across different regions. Sichuan, 1 of the 10 regions with the

A

Chronic phlegm with/without AFO (LLN) - Age adjusted

Females
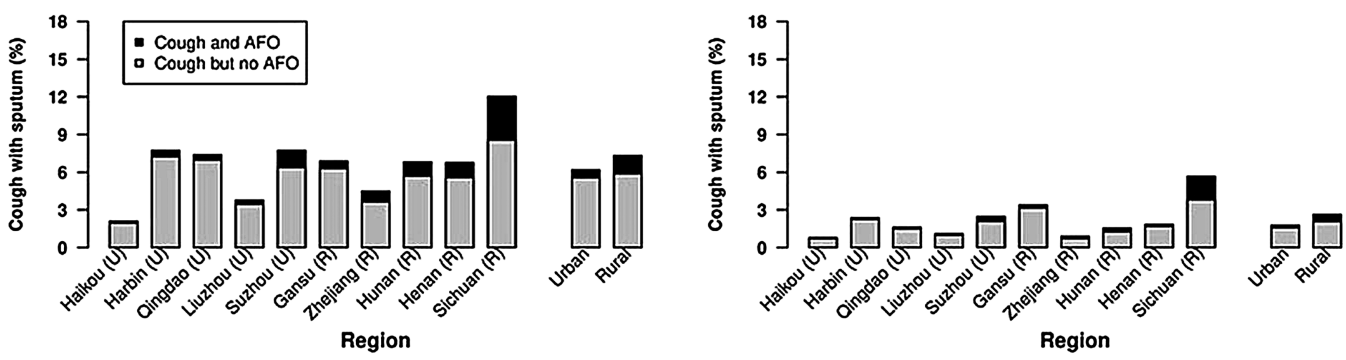

Breathlessness with/without AFO (LLN) - Age adjusted
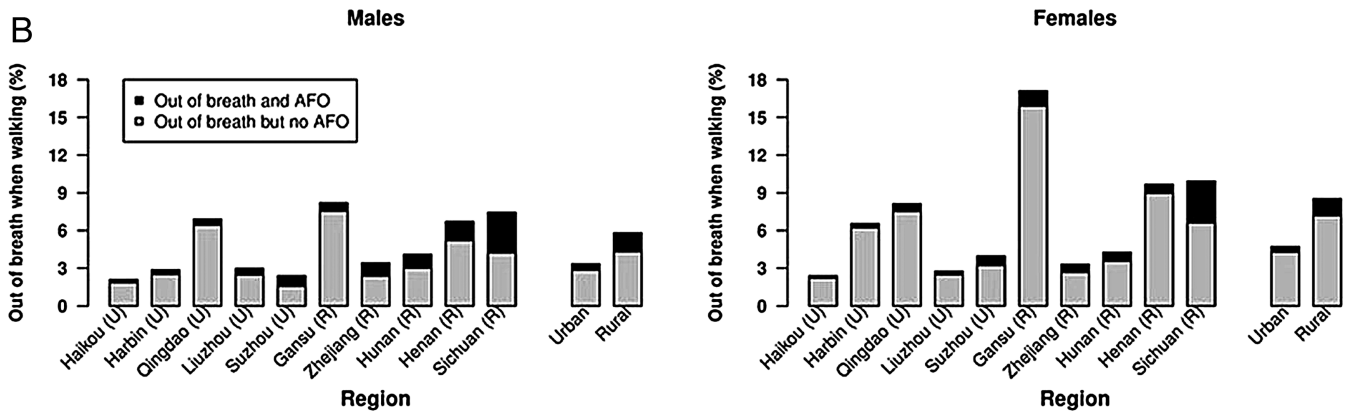

Figure 4 Prevalence of age-adjusted (A) chronic cough and phlegm with air flow obstruction (lower limit of normal, LLN), and (B) breathlessness with airflow obstruction (LLN). 
highest prevalence of $\mathrm{AFO}$, was included because of higher mortality rate from COPD reported in our previous studies. ${ }^{15}$ The study area in Sichuan is situated in a valley and environmental conditions such as temperature inversion could play a role in the prevalence of $\mathrm{AFO}$ as it is related to pollution levels. Currently, data on genetic biomarkers for a number of health outcomes, including respiratory health, is being studied in this population that could possibly explain some of the variations in the prevalence of AFO in different regions. The prevalence of asthma in our sampling population was low and similar to previous findings ${ }^{16}$; lower diagnosed asthma prevalence in China could be due to poor diagnostic facilities making it difficult to differentiate between obstructive lung diseases.

As in previous studies, ${ }^{5}$ we found that men had higher prevalence of AFO than women, probably due to high smoking prevalence in Chinese men. The higher prevalence observed among rural women could be due to greater exposure to environmental pollutants including household air pollution while cooking food using solid fuel, low socioeconomic status or lung infections early in life.

In the present study we reported AFO results based on fixed forced expiratory ratio and LLN of forced expiratory ratio so that comparison with previous studies with different diagnostic criteria could be made. The AFO prevalence based on LLN criteria using GLI reference equations was higher than the forced expiratory ratio criteria. This could be due to higher cut-off values for forced expiratory ratio in the Chinese population with age under 60. Previous papers have reported higher falsepositive in the elderly population when using the fixed ratio criteria as it lacks specificity, but using the GLI reference equations to some extent overcomes the problem, although it needs to be validated independently. ${ }^{17} 18$

The extent of underdiagnosis and overdiagnosis of $\mathrm{AFO}$ in the present study population is much greater than that reported previously in western countries. ${ }^{19-24}$ The spirometry-based prevalence of AFO is much greater than self-reported prior physician-diagnosed $\mathrm{CB} / \mathrm{E}$. In China, the majority of COPD diagnoses are based on clinical examination and measurement of lung function is not common, particularly, in rural health clinics. ${ }^{25}$ Our study showed that younger participants, particularly those who are asymptomatic and current smokers and have middle-household income, are relatively more likely to be underdiagnosed. When subgrouped for severity of AFO based on the percent predicted $\mathrm{FEV}_{1}$, the majority of participants had either moderate or severe AFO suggesting that underdiagnosis might be more likely. Validation of this finding would be important as these participants are at a higher risk of developing COPD and early preventive action such as smoking cessation in these groups would be expected to gain the most long-term benefit.

Those with a smoking history and presence of respiratory symptoms were more likely to be overdiagnosed for
AFO. Similar findings have also been reported in other studies. A recent study in the $\mathrm{USA}^{26}$ found that $42.5 \%$ of those diagnosed with COPD were false positive with no airway obstruction, with most of the false-positive diagnosis seen among smokers with presence of respiratory symptoms. New GOLD guidelines recommend that spirometry should be one of the essential criteria for the clinical diagnosis and management of COPD ${ }^{27} 28$ among those reporting chronic productive cough or sputum production, dyspnoea and exposure to risk factors. Although spirometry is more reproducible and has greater sensitivity and specificity compared with peak expiratory flow, its use is not that frequent in many lowincome and middle-income countries, particularly in rural areas. $^{29}$ The relatively low prevalence of selfreported $\mathrm{CB} / \mathrm{E}$ in our study could be due to lack of awareness of the problem in the participants and also lack of adequate health facilities in proximity where the participants dwell. Further, recall bias could also not be excluded as a cause of the observed low proportion of diagnosed participants with AFO.

In spite of a large sample size and wide geographical locations covered, our study has some limitations. First, we did not administer a bronchodilator as part of the spirometry procedure, and hence no postbronchodilator lung function measurement was carried out. Postbronchodilator forced expiratory ratio lower than LLN or 0.70 is suggested to confirm persistent air flow limitation and thus the presence of COPD in the latest ERS/ATS and GOLD guidelines. ${ }^{28} 30$ This means the AFO observed in our study could be either COPD or asthma related, therefore the prevalence may be somewhat overestimated. Although postbronchodilator is often used to identify patients with COPD and the course of treatment, its use to differentiate from asthma could be influenced by the day of testing, the baseline lung function before the delivery of testing and also the number of drugs given to test. ${ }^{31}$ Second, the instrument we used at baseline did not give us the spirogram and thus incomplete exhalation could not be ruled out completely although every effort was made to explain to the participants to blow out as long as possible. A reduction in FVC due to incomplete exhalation could lead to underestimation of AFO and also could be the reason for higher prevalence of restrictive lung disease observed in our population where we used a modified definition, considering that we did not collect total lung capacity data that is usually required to define the restrictive lung disease. Third, we did not collect exacerbation history data at baseline and used GLI predictive equations ${ }^{12}$ based on the latest predictive equations for north China and southeast Asia where bias due to internal migration from south to the north cannot be completely excluded. Although most of the participants in our study were Han Chinese, some degree of misclassification of grade of AFO as classified by GOLD criteria may have occurred.

In summary, this extremely large study provides good evidence about the burden of AFO in adult Chinese 
men and women. Owing to the lack of use of spirometry for diagnosing AFO in routine clinical practice, a high proportion of such patients were not identified previously. Even among those with prior diagnosis of COPD, two-thirds lacked long-term treatment. Although a number of socioeconomic and lifestyle factors were associated with poor detection and treatment, a large proportion of regional variation remained unexplained. These findings highlight major respiratory health problems in China that need immediate attention to carry out appropriate interventions for optimal disease management as well as to develop the prevention strategies to be implemented in order to improve the current and future respiratory health in the Chinese population.

\section{Author affiliations}

${ }^{1}$ Nuffield Department of Population, University of Oxford, Oxford, UK

${ }^{2}$ School of Public Health, Peking University Health Science Center, Beijing,

People's Republic of China

${ }^{3}$ Chinese Academy of Medical Sciences, Dong Cheng District, Beijing,

People's Republic of China

${ }^{4}$ China National Center for Food Safety Risk Assessment, Beijing, People's Republic of China

${ }^{5}$ Tongxiang Center for Disease Control, Tongxiang, Zhejiang, People's

Republic of China

${ }^{6}$ Liuzhou Center for Disease Control, Liuzhou, Guangxi, People's Republic of China

${ }^{7}$ Suzhou Center for Disease Control, Suzhou, Jiangsu, People's Republic of China

${ }^{8}$ Pengzhou Center for Disease Control, Pengzhou, Sichuan, People's Republic of China

${ }^{9}$ Henan Center for Disease Control, Zhengzhou, Henan, People's Republic of China

${ }^{10}$ Worldwide Epidemiology, GlaxoSmithKline R\&D, Uxbridge, UK

Acknowledgements The authors would like to thank Judith Mackay in Hong Kong; Yu Wang, Gonghuan Yang, Zhengfu Qiang, Lin Feng, Maigen Zhou, Wenhua Zhao and Yan Zhang in China Centres for Disease Control and Prevention (CDC); Lingzhi Kong, Xiucheng Yu and Kun Li in the Chinese Ministry of Health and Yiping Chen, Sarah Clark, Martin Radley, Mike Hill, Hongchao Pan and Jill Boreham in the CTSU, Oxford, for assisting with the design, planning, organisation and conduct of the study. The most important acknowledgement is to the participants in the study and the members of the survey teams in each of the 10 regional centres, as well as to the project development and management teams based at Beijing, Oxford and the 10 regional centres. ZC and $\mathrm{RC}$ acknowledge support from the BHF Centre of Research Excellence, Oxford.

Collaborators Members of China Kadoorie Biobank collaborative group, (A) International Steering Committee. Liming Li, Zhengming Chen, Junshi Chen, Rory Collins, Richard Peto. (B) Study Coordinating Centres, International Co-ordinating Centre, Oxford: Zhengming Chen, Garry Lancaster, Xiaoming Yang, Alex Williams, Margaret Smith, Ling Yang, Yumei Chang, Iona Millwood, Yiping Chen, Sarah Lewington, Sam Sansome, Robin Walters, Om Kurmi, National Co-ordinating Centre, Beijing: Yu Guo, Zheng Bian, Can Hou, Yunlong Tan, Zheng Wang, Xin Cai, Huiyan Zhou, Xuguan Chen, Regional Co-ordinating Centres, 10 areas in China: Qingdao Qingdao Centre for Disease Control: Zengchang Pang, Shanpeng Li, Shaojie Wang. Licang Centre for Disease Control: Silu Iv. Heilongjiang Provincial Centre for Disease Control: Zhonghou Zhao, Shumei Liu, Zhigang Pang. Nangang Centre for Disease Control: Liqiu Yang, Hui He, Bo Yu. Hainan Provincial Centre for Disease Control: Shanqing Wang, Hongmei Wang. Meilan Centre for Disease Control: Chunxing Chen, Xiangyang Zheng. Jiangsu Provincial Centre for Disease Control: Xiaoshu Hu, Minghao Zhou, Ming Wu, Ran Tao. Suzhou Centre for Disease Control: Yeyuan Wang, Yihe Hu, Liangcai Ma. Wuzhong Centre for Disease Control: Renxian Zhou. Guangxi Provincial Centre for Disease Control: Zhenzhu Tang, Naying Chen, Ying Huang. Liuzhou Centre for Disease Control:
Mingqiang Li, Zhigao Gan, Jinhuai Meng, Jingxin Qin. Sichuan Provincial Centre for Disease Control: Xianping Wu, Ningmei Zhang. Pengzhou Centre for Disease Control: Guojin Luo, Xiangsan Que, Xiaofang Chen. Gansu Provincial Centre for Disease Control: Pengfei Ge, Xiaolan Ren, Caixia Dong. Maiji Centre for Disease Control: Hui Zhang, Enke Mao, Zhongxiao Li. Henan Provincial Centre for Disease Control: Gang Zhou, Shixian Feng. Huixian Centre for Disease Control: Yulian Gao,Tianyou He, Li Jiang, Huarong Sun. Zhejiang Provincial Centre for Disease Control: Min Yu, Danting Su, Feng Lu. Tongxiang Centre for Disease Control: Yijian Qian, Kunxiang Shi, Yabin Han, Lingli Chen. Hunan Provincial Centre for Disease Control: Guangchun Li, Huilin Liu, LI Yin. Liuyang Centre for Disease Control: Youping Xiong, Zhongwen Tan, Weifang Jia.

Contributors ZC, LL, RP and RC were involved in the concept, design of the study and reviewing the manuscript. OK was involved in the analysis plan, preparing the first draft and editing of the manuscript. MS and MA were involved in the analysis plan, analysis of the data and reviewing the manuscript. JC, YG, YH, JQ, GX, JW, ZB and GZ were involved in the supervision of the data collection, data monitoring and reviewing the manuscript. $\mathrm{KD}$ was involved in the analysis plan and reviewing the manuscript. ZC is the $\mathrm{PI}$ and also was responsible for overall supervision of the project.

Funding The baseline survey and first resurvey in China were supported by a research grant from the Kadoorie Charitable Foundation in Hong Kong; follow-up of the project during 2009-2014 is supported by the Wellcome Trust in the UK (grant 088158/Z/09/Z), and the National Key Technology Research and Development Program in the 12th Five-Year Plan, Ministry of Science and Technology, China; the Clinical Trial Service Unit and Epidemiological Studies Unit (CTSU) at Oxford University also receives core funding for it from the UK Medical Research Council, the British Heart Foundation, and Cancer Research UK. Support for the present respiratory study was provided by GlaxoSmithKline (WEUKBRE5848).

Competing interests $\mathrm{KD}$ is employed by GlaxoSmithKline.

Ethics approval Central ethics approvals were obtained from Oxford University, the China National Centres for Disease Control and Prevention (CDC) and local ethics approvals from institutional research boards at the local CDCs in the 10 regions

Provenance and peer review Not commissioned; externally peer reviewed.

Data sharing statement No additional data are available.

Open Access This is an Open Access article distributed in accordance with the Creative Commons Attribution Non Commercial (CC BY-NC 3.0) license, which permits others to distribute, remix, adapt, build upon this work noncommercially, and license their derivative works on different terms, provided the original work is properly cited and the use is non-commercial. See: http:// creativecommons.org/licenses/by-nc/3.0/

\section{REFERENCES}

1. World Health Organisation. The global burden of disease: update 2004. Geneva: World Health Organisation, 2008. http://www.who.int/ healthinfo/global_burden_disease/GBD_report_2004update_full.pdf (accessed 29 Jan 2013).

2. Mathers CD, Loncar D. Projections of global mortality and burden of disease from 2002 to 2030. PLoS Med 2006;3:e442.

3. World Health Organisation. Disease and injury country estimates, 2008. Global health observatory data repository. World Health Organisation. http://apps.who.int/gho/data/node.main.1004 (accessed 27 Jan 2014).

4. World Health Organisation. Death and DALY estimates for 2004 by cause for countries: global health observatory data repository. World Health Organisation. http://apps.who.int/gho/data/node.main.1008 (accessed 27 Jan 2014).

5. Zhong N, Wang C, Yao W, et al. Prevalence of chronic obstructive pulmonary disease in China: a large, population-based survey. Am J Respir Crit Care Med 2007;176:753-60.

6. Xu F, Yin X, Zhang M, et al. Prevalence of physician-diagnosed COPD and its association with smoking among urban and rural residents in regional mainland China. Chest 2005;128:2818-23. 
7. Buist AS, McBurnie MA, Vollmer WM, et al. International variation in the prevalence of COPD (the BOLD Study): a population-based prevalence study. Lancet 2007;370:741-50.

8. Chen Z, Chen J, Collins R, et al. China Kadoorie Biobank of 0.5 million people: survey methods, baseline characteristics and long-term follow-up. Int J Epidemiol 2011;40:1652-66.

9. Fang X, Wang X, Bai C. COPD in China: the burden and importance of proper management. Chest 2011;139:920-9.

10. Chen Z, Lee L, Chen J, et al. Cohort profile: the Kadoorie Study of Chronic Disease in China (KSCDC). Int J Epidemiol 2005;34:1243-9.

11. American Thoracic Society. Standardization of spirometry, 1994 update. Am J Respir Crit Care Med 1995;152:1107-36.

12. Quanjer PH, Stanojevic S, Cole TJ, et al. Multi-ethnic reference values for spirometry for the 3-95-yr age range: the global lung function 2012 equations. Eur Respir J 2012;40:1324-43.

13. Miller MR, Quanjer PH, Swanney MP, et al. Interpreting lung function data using $80 \%$ predicted and fixed thresholds misclassifies more than $20 \%$ of patients. Chest 2011;139:52-9.

14. Miller MR, Crapo R, Hankinson J, et al. General considerations for lung function testing. Eur Respir J 2005;26:153-61.

15. Liang BM, Xu ZB, Yi Q, et al. Association of chronic obstructive pulmonary disease with coronary artery disease. Chin Med $J$ 2013;126:3205-8.

16. Chan-Yeung M, Zhan LX, Tu DH, et al. The prevalence of asthma and asthma-like symptoms among adults in rural Beijing, China. Eur Respir J 2002;19:853-8.

17. Pellegrino R, Viegi G, Brusasco V, et al. Interpretative strategies for lung function tests. Eur Respir J 2005;26:948-68.

18. Vollmer WM, Gislason T, Burney $P$, et al. Comparison of spirometry criteria for the diagnosis of COPD: results from the BOLD study. Eur Respir J 2009;34:588-97.

19. Halbert RJ, Natoli JL, Gano A, et al. Global burden of COPD: systematic review and meta-analysis. Eur Respir J 2006; 28:523-32.

20. Mannino DM, Buist AS. Global burden of COPD: risk factors, prevalence, and future trends. Lancet 2007;370:765-73.
21. Buist AS, Vollmer WM, McBurnie MA. Worldwide burden of COPD in high- and low-income countries. Part I. The burden of obstructive lung disease (BOLD) initiative. Int J Tuberc Lung Dis 2008;12:703-8.

22. Walters JA, Walters EH, Nelson M, et al. Factors associated with misdiagnosis of COPD in primary care. Prim Care Respir $J$ 2011;20:396-402

23. Shahab L, Jarvis MJ, Britton J, et al. Prevalence, diagnosis and relation to tobacco dependence of chronic obstructive pulmonary disease in a nationally representative population sample. Thorax 2006;61:1043-7.

24. Miravitlles M, Soriano JB, Garcia-Rio F, et al. Prevalence of COPD in Spain: impact of undiagnosed COPD on quality of life and daily life activities. Thorax 2009;64:863-8.

25. Lu M, Yao WZ, Zhong NS, et al. Asymptomatic patients of chronic obstructive pulmonary disease in China. Chin Med J (Engl) 2010;123:1494-9.

26. Christian G, Allen D, Magdi HA. Misdiagnosis and mistreatment of chronic obstructive pulmonary disease in an underserved patient population. D32 chronic obstructive pulmonary disease: worldwide epidemiology. American Thoracic Society, 2013:A5506-A.

27. Vestbo J, Hurd SS, Rodriguez-Roisin R. The 2011 revision of the global strategy for the diagnosis, management and prevention of COPD (GOLD)—why and what? Clin Respir J 2012;6:208-14.

28. Vestbo J, Hurd SS, Agusti AG, et al. Global strategy for the diagnosis, management, and prevention of chronic obstructive pulmonary disease: GOLD executive summary. Am J Respir Crit Care Med 2013;187:347-65.

29. Gershon AS, Victor JC, Guan J, et al. Pulmonary function testing in the diagnosis of asthma: a population study. Chest 2012;141:1190-6.

30. Wanger J, Clausen JL, Coates A, et al. Standardisation of the measurement of lung volumes. Eur Respir J 2005;26:511-22.

31. Calverley PMA, Albert P, Walker PP. Bronchodilator reversibility in chronic obstructive pulmonary disease: use and limitations. Lancet Respir Med 2013;1:564-3. 


\section{Correction}

Kurmi OP, Li L, Smith M, et al. Regional variations in the prevalence and misdiagnosis of air flow obstruction in China: baseline results from a prospective cohort of the China Kadoorie Biobank (CKB). BMJ Open Resp Res 2014;1:e000025. doi:10.1136/bmjresp-2014-000025

The author Zhenming Chen has been corrected to read Zhengming Chen.

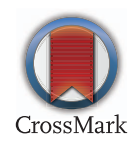

BMJ Open Resp Res 2014;0:e000025corr1. doi:10.1136/bmjresp-2014-000025corr1 